\title{
Expertise et censures : les aventures d'un "sociologue national"
}

\section{Diouldé Laya}

\section{(2) OpenEdition}

\section{Journals}

Édition électronique

URL : http://journals.openedition.org/apad/1551

DOI : 10.4000/apad.1551

ISSN : 1950-6929

Éditeur

LIT Verlag

\section{Édition imprimée}

Date de publication : 1 juin 1995

\section{Référence électronique}

Diouldé Laya, «Expertise et censures : les aventures d'un "sociologue national" », Bulletin de I'APAD [En ligne], 9 | 1995, mis en ligne le 26 juillet 2007, consulté le 07 septembre 2020. URL : http:// journals.openedition.org/apad/1551; DOI : https://doi.org/10.4000/apad.1551

Ce document a été généré automatiquement le 7 septembre 2020

Bulletin de l'APAD 


\title{
Expertise et censures : les aventures d'un "sociologue national" 1
}

\author{
Diouldé Laya
}

Introduction

1 Ce texte rend compte de mon expérience d'étudiant (inscrit en licence de sociologie dès sa création, en 1958-59, dans la dix-huitième université française, c'est-à-dire Dakar) puis de mon accès au statut de "sociologue national". J'étais fier d'être "sociologue national", à un moment où il fallait tout bâtir dans le domaine de la recherche sociologique.

2 Je vais donc réfléchir sur mes activités au cours de mes études, puis des années 1965-80 correspondant à une partie de ma vie professionnelle, tout en me limitant aux travaux commandés (évaluations, enquêtes préliminaires) et à leurs divers aspects (relations avec les services administratifs et les organisations internationales, rédaction du rapport, etc). Il s'en dégagera la ligne suivie par ce jeune chercheur que j'étais, dans sa pratique de l'expertise.

RepèresLe banc

3 A l'Université de Dakar, nous avions eu la chance de passer deux semaines chez les Diolas de Casamance, sous la direction de Louis-Vincent Thomas, et d'aller trouver sur le terrain, au moins deux fois, Abdoulaye Bara Diop faisant son enquête sur les migrations toucouleur à Dakar. Cela devait se passer en 1958-59 ou 1959-60. Par contre les deux dernières années m'ont semblé très difficiles.

4 J'ai suivi les cours de linguistique africaine. J'ai été, il me semble cette année-là, sollicité comme informateur pour aider le Pr. Greenberg à vérifier certains mots : comprenant un peu bambara, fulfulde, hausa et songhay, j'ai gardé le sentiment que le choix de certains mots suscitait des interrogations, mais un étudiant doit toujours être correct. Depuis cette date, la littérature sociologique me révolte toujours moins que la myopie des linguistes. Samba N'Diaye et moi avons été chargés, en 1962, de remplir un questionnaire du Pro Georges Balandier sur le temps (étude menée, nous avait-on expliqué, à la demande d'une célèbre marque de montre). 
5 Très démoralisé par une série de mesures antidémocratiques qui aboutiront à la dissolution de l'Union Générale des Etudiants d'Afrique occidentale, UGEAO, je rejoignis, en Juillet 1962, Niamey où le plan triennal intérimaire 1961-63 avait été adopté. Je voulais préparer, à partir des enquêtes sur le terrain, un diplôme d'études supérieures puis aller à Paris me spécialiser en démographie.

\section{Le Centre Nigérien}

6 Je demandai mon affectation à l'Institut du Folklore et d'Art Nigériens - Centre Nigérien de Recherches en Sciences Humaines, IFAN-CNRSH de Niamey. Le Ministère de l'Education Nationale décida de me garder à la Commission Nationale de l'UNESCO, récemment créée et dont le secrétariat était provisoirement assuré par un expert de cette organisation. Sans penser aux vacances, je me mis au travail, en Août ou Septembre de la même année. En Novembre ou Décembre, à mon retour de la $12 \mathrm{e}$ session de la Conférence Générale de l'UNESCO, je fus chargé de diriger la Commission Nationale, et on me confia en outre des responsabilités concernant les questions de recherche et de culture. Les Ministres sous lesquels j'ai servi m'ont étonné à la confiance totale qu'ils m'ont accordée.

7 En Décembre 1964, je devins l'homologue de l'expert des Nations Unies qui avait le titre de Conseiller pour la Recherche en Développement Communautaire et Ressources Humaines, le Dr. Charles Pidoux. Le même mois, un bureau nous fut affecté dans les nouveaux locaux du CNRSH. Cette institution était en effet le lieu où se côtoyaient philosophes devenus ethnologues ou anthropologues, ethnologues et anthropologues devenus sociologues, sociologues devenus socio-économistes parce que la sociologie tentait de se tailler un champ propre, en se dégageant de la philosophie, l'économie politique (science économique), et surtout l'envahissante anthropologie. D'après les rapports d'activités de 1962 et 1962-65, le programme du Centre défini par le Plan triennal intérimaire comprenait deux axes :

- étude fondamentale sur les groupes ethniques, les principales ethnies du Niger ;

- étude de problèmes prioritaires liés au développement.

Jean Rouch en était le directeur scientifique. Suzanne Bernus en assurait le secrétariat avec compétence, dévouement et autorité : grâce à elle et beaucoup d'autres, mais elle en tout premier lieu, le Centre de Niamey connut un rayonnement incontestable. En effet, pour Jean et Suzy, le Centre devrait faire "la recherche d'intérêt national, mais de valeur internationale". Il y avait avec nous d'autres chercheurs français : Raulin, Piault, Raynaut, Echard, Olivier de Sardan, etc. Pour ce qui est du Niger, outre Boubou Hama, il y avait le Dr Kélétigui Abdourahmane Mariko, vétérinaire, qui avait suivi un stage de planification à l'Université de Dakar pendant que j'y étais encore.

Sous la présidence de Jean Rouch, une réunion examina, les 29, 30 et 31 Décembre 1964 au Centre, les problèmes de la coordination des études et des recherches en sciences sociales appliquées au développement: le Dr. Pidoux et moi y avons assisté. Un an après, on annonça une réunion prévue du 4 au 8 Novembre 1965 sur la coordination de la recherche scientifique au Niger, avec la participation d'un consultant de UNESCO.

Mon intégration a été facilitée par Boubou Hama, Jean Rouch, le Dr. Pidoux, et le Dr. Mariko. Je distingue deux périodes : 1965-72 et 1973-80. J'avais dû renoncer à une enquête sur la rumeur en 1964-65 (époque de la "subversion" par le Sawaba) et sur les conditions et conséquences de l'expulsion des Africains en 1968 ("expulsés de Busia", c'est-à-dire du Ghana). Mais la sécheresse de 1973 m'imposera d'étudier les conditions 
d'existence des agriculteurs et des éleveurs pour les aider dans leur lutte quotidienne : le coup d'état militaire d'Avril 1974 a été directement favorisé par la sécheresse, le problème du prix de l'uranium étant rarement évoqué, comme l'exige l'art de diriger la cité. En tout cas, je ne pouvais plus avoir le monopole, car la seconde génération de sociologues faisait son entrée, et en force.

1965-1972 : Sociologue national

11 J'ai écrit quelque part que même Fatoumata Agnès Diaroumèye (Mme Diarra, sociologue, spécialiste de psychologie sociale, actuellement directrice du Bureau du MULPOC/CEA, à Yaoundé, Cameroun), qui l'aurait pu, ne m'a pas ravi ce glorieux titre.

Relations

12 Excellentes avec les services nationaux et les organisations internationales, les relations ont été empreintes d'une grande amitié avec les divers experts nigériens. Quant aux spécialistes venus de l'extérieur, pour près du quart d'entre eux, seule l'ignorance l'a cédé au complexe de supériorité.

Relations avec les services et organisations internationales

13 Mes rapports furent très étroits avec le Commissariat Général au Développement. C'est en effet lui qui déterminait l'ordre de priorité des études et recherches liées au développement après en avoir négocié le financement. Pratiquement toutes les enquêtes préliminaires de 1965 et 1966 furent réalistes à sa demande, par l'intermédiaire du Service du Génie Rural rattaché au Ministère de l'Economie Rurale, et dirigé par Francois Gadelle, un polytechnicien parfaitement à l'aise avec les sciences sociales et humaines. Je dois ajouter que Gabriel Mayaki et Ali Seyni, du même service, m'ont soutenu, défendu, et amené ou accompagné sur le terrain afin de mieux discuter. Mes premières enquêtes en 1965 et 1966 firent apparaître une concentration des terres entre les mains de "féodaux" et de "propriétaires fonciers". En fait, celles menées par Raulin en 1961/63 avaient attiré l'attention sur le nombre de champs possédés par deux personnalités politiques de premier plan; le Dr. K.A. Mariko est très informé et documenté sur le sujet. Je maintiens que la taille moyenne de l'exploitation, le recours quasi exclusif à l'énergie humaine, la très mauvaise association actuelle entre l'agriculture et l'élevage ne peuvent garantir une production agricole susceptible de couvrir les besoins de la population.

14 Le Ministère de la Jeunesse et des Sports comptait un certain nombre de cadres compétents, dévoués, et très sympathiques. J'ai coopéré loyalement avec eux. Ainsi, Mounkaïla Hassane m'avait demandé en 1963 de faire une conférence à l'occasion du centenaire de la naissance du baron Pierre de Coubertin, rénovateur des Jeux Olympiques, donc de replonger dans mon univers antique. Pour obtenir le certificat d'aptitude à l'inspectorat de la jeunesse et des sports, il a présenté en 1970 un mémoire intitulé : "Pour une animation sportive et sociale au Niger". En 1971, il s'est associé au sondage sur les besoins et aspirations des jeunes, financé par l'UNICEF.

15 Au Ministère de la Promotion Humaine, responsable de l'élaboration du rapport du Niger au forum "Famille-Enfance Jeunesse-Développement", organisé à Lomé en 1972, travaillait feu Moussa Mamadou, dont j'estimais, dès 1963, la présence très souhaitable dans les services de l'animation. Pour obtenir le diplôme de l'Ecole des Hautes Etudes en Sciences Sociales, il a présenté, en 1978, une étude intitulée : "Analyse d'un projet de développement rural au Niger. D'un projet de mise en valeur et sa mise en échec (le cas du Dallol Maouri en République du Niger)". 
Nationale de Sécurité Sociale, CNSS. Une discussion entre lui et le Dr. Pidoux permit de fixer le cadre et les objectifs de l'étude sur "la situation des personnes âgées de 50 ans et plus sur le territoire de la Commune de Niamey". L'enquête fut achevée en Novembre 1967, l'analyse des données recueillies ayant été laissée au soin de l'expert.

21 Nos contacts avec l'Organisation des Nations Unies pour l'Alimentation et l'Agriculture (FAO) datent de 1965, lors de la préparation de l'enquête préliminaire sur la cuvette de Kirkissoy, dans la vallée du fleuve Niger. En Mars 1966, eut lieu notre second séjour sur le terrain du projet "Développement de la production animale et des ressources en eau dans l'est du Niger".

La particularité, en ce qui concerne l'UNICEF, est que la plupart des directeurs régionaux qui s'étaient succédés à Abidjan étaient des connaissances, sinon des amis. En même temps, il était de mon devoir de participer, dans la mesure du possible, à la réalisation de leur programme. Le facteur personnel ne doit pas être sous-estimé, 
surtout qu'il a trouvé un terrain très favorable du fait de l'esprit de coopération manifesté par les Ministères de la Jeunesse et de la Promotion Humaine.

Il va de soi que le statut d'homologue d'un expert de l'assistance technique des Nations Unies est une excellente référence dans les relations avec les organisations du système des Nations Unies.

Experts

24 Fondamentalement, je ne vois pas de différence entre un administrateur nous accusant de "créer" les classes sociales et le responsable de service nous prêtant l'intention de lui faire perdre sa place. Tous deux sont choqués par le caractère objectif que nous voulons donner à nos investigations.

25 J'ai eu plusieurs séances de travail avec les cadres du Service du Génie Rural et de la Direction des Travaux Publics: elles ont porté sur le contenu exact du projet, l'explicitation de tel ou tel aspect de celui-ci, le rapport présenté, etc. L'un des objectifs du Conseil National de la Recherche Scientifique et Technique était de favoriser l'amorce d'un dialogue permanent entre divers spécialistes, entre développeurs et décideurs : je ne puis dire s'il y a changement, mais je ne le pense pas.

Quelques Français avaient le complexe de supériorité. L'un voulait absolument se faire reconnaître comme directeur d'une base de recherche se réduisant au maximum à deux personnes: un jeune chercheur français et un excellent collaborateur technique nigérien. Il anticipait ainsi sur la création future de bases à travers le Niger. Celles d'Agadès et de Maradi sont aujourd'hui opérationnelles, même si je n'avais pas réussi à en implanter à Zinder, Tahoua, Diffa et surtout Tillabéri pour bien montrer que Rouch n'avait pas épuisé la recherche. Pour l'autre, on ne devenait digne de diriger le Centre Nigérien que si l'on était archéologue, et si l'on avait parcouru au moins deux fois l'Air ; or, je n'étais pas encore directeur, et je n'avais aucune possibilité immédiate de bénéficier d'une telle formation. De plus, lui oubliait qu'il était venu au Centre dans une Land-Rover climatisée mise à sa disposition par le Niger qui y avait déjà affecté à temps partiel un jeune archéologue.

27 J'ai contribué petit à petit à réorienter les projets ou le terrain des chercheurs venant de l'Amérique et de l'Europe, de façon à constituer une documentation variée. En effet, les Touaregs et les Peuls intéressaient trop les Européens, La plupart donnaient comme prétexte que Jean Rouch avait tout dit sur l'ouest Voilà pourquoi le rapport sur les activités du Centre (Octobre 1969 - 31 Décembre 1971) précise (pp.33-46) :

- le pays d'origine et la discipline des missions accueillies de 1962 à 1971 ;

- par année, entre le 1/10/1969 et le 31/12/71, la liste des missions accueillies et de leurs projets d'étude.

$\mathrm{Au}$ vu de ces données, j'avais estimé, en ma qualité de directeur du Centre (car j'avais été nommé en Mars 1970) qu'il fallait réduire, sinon interdire les recherches sur les Touaregs et les Peuls devenus l'objet privilégié de l'ethnologie : la sécheresse de 1973 n'arrangea rien. D'ailleurs, l'ignorance était réelle : relative chez quelques chercheurs, elle pouvait être absolue chez certains experts. Tel spécialiste venu travailler sur les Touaregs ignore l'existence, au Niger, d'autres pasteurs, peuls en particulier. Un groupe d'étudiants polonais, lors d'une visite au Centre, me demanda combien de tribus le Niger comptait. Je pouvais parfaitement admettre l'ignorance du fait de l'option politique du Gouvernement du Niger, mais pas ce degré d'impréparation en Pologne.

Recherche 
Je m'étais arrangé pour ne pas rentrer par crainte d'être affecté dans l'enseignement. En effet, dans un lycée, j'aurais peut-être assumé un cours de philosophie (matière que je n'aimais pas, puisque je ne la comprenais pas), bien que le programme du second cycle fasse un peu de place aux sciences sociales. Puis un jour, Inoussa Ousseini, animateur d'un Ciné-club dont les membres étaient presque tous élèves de l'actuel Lycée Issa Korombé, eut l'idée de m'associer à un débat sur le film de Jean Rouch "Les maitres-fous". Pour les dégoûter, je leur dis avec tout le sérieux nécessaire que certains de mes camarades migrants m'avaient informé de ce que Jean avait osé montrer au cinéma. La virulence des propos tenus par certains résonne encore dans mes oreilles, mais je ne suis pas sûr que Inoussa leur ait présenté un film qui ne fasse pas frémir d'indignation. Et je me suis toujours senti malheureux d'avoir à assurer une série de cours (transformés en débats) dans quelques établissements de formation professionnelle (en commençant par l'Ecole Nationale de la Santé Publique de Niamey) sur les "problèmes humains du développement". Une telle formulation était tout de même plus explicite que "étude des structures mentales", chère aux spécialistes d'une certaine science sociale, et à quelques experts. Le Dr. Pidoux m'a aidé à combattre tout un courant de pensée, et à mieux concevoir ma participation à la formation de ces cadres. J'étais soulagé d'en être déchargé par Issaka Doulaye, Garba Hima et Michel Keita, tous bénéficiaires d'une meilleure formation et prêts à m'aider.

Pour les gens, le sociologue était capable de réfléchir sur des questions complexes futiles ou graves, donc sur n'importe quoi, et en fin de compte, sur rien de précis. Incontestablement, on pouvait jouir d'une bonne marge de manœuvre. Mais il faut faire très attention. Le Ministère, lui, savait me faire travailler, tout en me laissant la liberté de faire de la recherche. Et mes premiers pas sur le terrain au Niger furent angoissants : je me rendis compte que le chercheur africain était aussi éloigné des langues et cultures nationales que d'une discipline faisant, en toute indépendance, ses premiers pas dans l'Europe occidentale capitaliste. L'ethnographie que je pratique en Afrique est ma sociologie, même si l'on préfère parler d'anthropologie. Hélas! Un jour, j'interprétais pour les riziculteurs de Koutoukalé les propos de Raulin. Il expliqua que la cuvette représentait une source de revenus, une richesse potentielle, disant "Cette cuvette, c'est de l'or"; je dis très fièrement : "farro wo, wura no". Aucun auditeur ne réagit. Il m'a fallu une semaine pour réaliser ce qui m'était arrivé : ma traduction littérale était un échantillon du discours de nègre blanc. Au sortir de l'université, on devra être modeste, et saisir toutes les occasions de faire des enquêtes. Dès au départ, j'ai bénéficié de la collaboration de Mamadou Ouattara dit Petit Touré, un chef enquêteur expérimenté. Et de 1965 à 1972, j'ai participé à la réalisation de, ou réalisé sept évaluations (1966, 1967, 1968, 1971), cinq enquêtes préliminaires $(1965,1966,1972)$.

Évaluations

31 Cela donne une moyenne de deux projets par an, mais la réalité est encore plus instructive. Les données de deux évaluations ("cité industrielle de Malbaza" en 1966, "situation des personnes âgées" en 1968) ont été recueillies et remises à deux experts de l'assistance technique des Nations Unies. Dans les deux cas, le travail avait été fait par Dr. Pidoux et moi. Nous étions au moins sûrs des informations fournies aux utilisateurs. Mais je n'ai pas retrouvé trace du compte-rendu de l'enquête de 1971 sur les "besoins et aspirations des jeunes", financée par l'UNICEF. Le rapport Enfance-Jeunesse-Famille-Développement présenté au forum de Lomé en 1972 y fait allusion et des jeunes avaient prévu, au Niger, une révolution avant l'an 2000. Lors du 
sondage sur "les problèmes de la zone industrielle de Niamey", réalisé en 1968 avec Suzanne Bernus, j'avais rencontré quelques vieux "entrepreneurs" ; n'ayant pas réussi à obtenir un entretien satisfaisant avec quelques autres personnes, j'avais laissé à Suzy le soin de tenter de les convaincre.

A la fin du premier trimestre de 1973, j'avais été sollicité pour évaluer la situation de la Société Nigérienne des Primeurs, SONIPRIM, de Niamey. La Société connaissait de grandes difficultés, et souffrait d'un mauvais fonctionnement L'enquête fut menée entre Avril et Juin par une équipe que dirigeait le même chef enquêteur. Je n'ai jamais rédigé le rapport, pour des raisons que je vais essayer de préciser. La première est que, tout près du périmètre de la Société, était située l'exploitation (marchant très bien) d'une personnalité que je rencontrais, sans le vouloir, deux fois au moins, non loin du lieu d'enquête. Je craignais donc des implications politiques directes. La seconde est que la Société était l'exemple d'une mauvaise réalisation dans le secteur de l'économie mixte : la société de transformation du mil, SOTRAMIL, servira de déversoir à mon amertume. La troisième est mon hostilité à l'égard des haricots verts, car un tel projet confisque les terres des paysans, puis transforme ceux-ci en ouvriers agricoles : c'est très exactement la prolétarisation; même si l'exportation des haricots verts rapporte des devises, de telles conditions sont politiquement inacceptables.

L'évaluation, en Mai 1971, de la situation dans la zone du projet "Développement de la production animale et des ressources en eau dans l'est du Niger" a été faite avec l'aide d'un collaborateur de choix. L'interprète de hausa est un historien originaire de la région, et passionné par les problèmes que vivent les pasteurs, André Salifou. Grâce à une bonne préparation à Zinder, aux discussions avec le Dr. Gouro, aux diverses données recueillies par interview enfin, le rapport présenté fut jugé satisfaisant, selon des échos parvenus longtemps après, du côté de la FAO. Aujourd'hui, je dois formuler trois remarques critiques sur ce rapport :

- excès de respect pour une structure traditionnelle (chefferie);

- excès de confiance dans l'administration "moderne" ;

- absence totale de référence à une alternative, par exemple la coopérative, ou l'association de pasteurs, soit parce que je n'avais pas bien digéré ce qui allait être publié sous le titre "La Voie peule", soit parce qu'il manquait la sécheresse.

Enquêtes préliminaires

L'étude de la situation dans les cuvettes de Koutoukalé et Kanna m'avait permis de réaliser un rêve : présenter un mémoire à partir de travail sur le terrain. L'enquête sur Kirkissoy est moins connue alors qu'elle m'a fait entrer en contact avec un expert de la FAO. Je me suis refusé pendant longtemps à aller à Karma et Koutoukalé, parce que l'aménagement n'avait pas été réalisé dans des délais "raisonnables". Je m'étais senti un peu comme un menteur. Mais cela est moins grave que le fait de n'avoir pas été présent lors du démarrage de l'aménagement. Ce qui traduit bien la place de l'expert qui fait seulement l'étude, et cesse d'exister. Car si j'avais pu suivre l'évolution de la situation, j'aurais beaucoup amélioré mes connaissances sur les sociétés africaines. C'est certainement la raison pour laquelle je n'ai pas rédigé le rapport à la fin d'une étude.

En 1966, après des discussions avec les services intéressés, je fis démarrer l'enquête préliminaire au projet de barrage de Diapenga. L'édification du barrage avait pour buts de :

- développer la pisciculture, 
- permettre la pratique de cultures d'irrigation,

- résoudre le problème de l'abreuvement des troupeaux pendant la saison sèche. recherche scientifique et technique. En fait, il manquait une politique de ce domaine, ainsi que les ressources humaines et financières nécessaires à sa mise en œuvre, bien que le Niger ait été représenté à toutes les assises de l'OUA et de UNESCO consacrées au 
problème. Il faut mentionner aussi les résistances de plusieurs organismes; les Africains n'avaient rien à dire au sujet des domaines réservés des instituts français ; les Nations Unies avaient envisagé de fournir un appui au CNRSH pour la recherche en développement. Ce projet n'eut aucune suite.

Cependant, deux secteurs méritent d'être mentionnés. Sous l'impulsion de Abdou Moumouni, et dans des conditions difficiles, l'ONERSOL réorganisé attira de jeunes chercheurs, et s'imposa par son programme et ses réalisations (chauffe-eaux, pompes et piles solaires, etc.). Soumana Idrissa réunit des données sur la mineuse d'épi, réapparue en 1966-68 à la suite d'une sécheresse réelle ; en même temps, il s'intéressa à l'environnement. Mounkaïla Garba s'engagea dans l'étude et la récolte de quelques plantes. Cet assaut contre le quasi monopole des sciences sociales et humaines était très salutaire. Mais, jouant sur le sous-entendu, certains continuaient à clamer "le Niger n'a pas besoin de philosophes". Or il avait besoin et a besoin de personnes qui pensent, et de manière autonome.

Pour permettre au CNRSH/IRSH de s'acquitter de sa mission, les relations de travail furent créées et maintenues avec les chercheurs et enseignants nigériens. Grâce au système d'affectation à temps partiel, un historien remplaça un autre. Surtout la participation aux recherches fut encouragée : je n'ai pas rencontré un refus de la part d'expert nigérien. Par contre, l'administration ne comprend toujours pas que l'on puisse, pour un certain temps, décharger un fonctionnaire dont la compétence peut contribuer à l'étude et à la résolution des problèmes du développement. Elle préférera donc l'expert non local.

45 J'ai eu des séances de travail enrichissantes avec Thérèse Maïga, et feu Idrissa Diawara. J'ai discuté avec ce dernier le questionnaire qu'il avait élaboré lors de la préparation du doctorat de 3e cycle. Au moins lui et moi avions reçu, après avril 1974, un titre de transport et des frais de séjour pour aller, en France, soutenir une telle thèse. J'avais bénéficié d'une telle facilité, et j'en avais informé Diawara. Peut-être faut-il attribuer cette décision à la présence, à la tête du Ministère de l'Education Nationale, du Général Henri Dupuis Yacouba. Peut-être aussi ai-je profité de la complaisance d'un nigérien formé à Kati au Mali ${ }^{3}$.

J'ai également examiné la traduction en hausa et en songhay des questionnaires sur les produits de la SOTRAMIL. Il y eut de très vives discussions avec Abdou Hamani et Hamidou Lailaba Kô. Voilà un exercice auquel nous nous livrons très rarement.

Accepter ou refuser les contrats de recherche au nom de l'Institut, déterminer la fraction du budget devant être attribuée à l'expert, à l'Institut, et à l'expert associé, modifier les termes de référence pour avoir une équipe multidisciplinaire, telles furent quelques unes des questions à résoudre. Peu à peu, ce qui était considéré comme une prédominance des recherches sociologiques agaça des collègues. Je ne comprenais pas pour quelle raison on s'opposa, en 1974, au départ de Michel Keita pour un stage à l'IDEP de Dakar. Malgré tout, grâce à moussa et lui, les petits déjeuners avaient été souvent gais.

Recherche Inoussa Ousseini et Michel Keïta étaient tous deux chargés de services scientifiques. Ce fut la période de l'épanouissement.

Évaluations 
En 1974, à la demande de la SOTRAMIL, Michel et moi tentons de mesurer l'acceptabilité des produits fabriqués. La Société, comme beaucoup d'entreprises créées dans les mêmes conditions, avait des problèmes, dont celui de l'écoulement des pâtes alimentaires, biscuits, couscous à base de mil. Après discussion avec les demandeurs, nous avons procédé à une enquête par sondage. Le rapport qui en résulte nous a semblé satisfaisant sur le plan scientifique; mais les produits n'étant pas très appréciés, leur réélaboration fut recommandée. Il est normal de penser à la transformation du mil. Si j'ai bonne mémoire, c'est d'abord la fabrication du whisky qui avait été envisagée. Le projet fut abandonné, si tant est qu'il ait été clairement exprimé. Même si des expériences étaient en cours au Sénégal (plus que le mimétisme, c'est le refus de la rationalisation qu'on peut identifier dans cette option), on aurait pu penser à autre chose que les pâtes alimentaires. Nous avons saisi l'occasion pour exprimer très clairement notre réserve sur un aspect de la politique de développement. En effet, le mil peut ne pas être disponible, du moins en quantité suffisante.

Enquêtes préliminaires

50 En 1975-76, sous la direction de Michel Keita, l'Institut s'est consacré à l'étude de Say. Une fois libérée de l'onchocercose, la région devait :

- accueillir des populations vivant dans les zones plus pauvres du fait de la sécheresse et de l'épuisement des sols (Zarmaganda, Kourfey),

- offrir aux troupeaux des points d'eau permanents et des pâturages.

Le schéma directeur de mise en valeur de l'arrondissement a servi de référence. L'étude fut menée par une équipe internationale (quatre nigériens, trois français), multidisciplinaire (agronomie, géographie, sociologie), et expérimentée (le plus novice avait fini son stage d'étudiant en agronomie, après avoir régulièrement collaboré avec l'Institut depuis l'Université). Ayant travaillé ensemble pendant plusieurs années, les membres ont mis au point le programme, défini la méthodologie et lancé l'étude, assistés d'un groupe d'enquêteurs. Celui qui avait guidé mes premiers pas en 1965 ne se trouvait plus avec moi : il avait décidé de ne pas travailler avec le responsable du Département de Socio-Economie, c'est-à-dire Michel Keïta, mon patron à moi aussi !

Les rapports ont été accompagnés de nombreux documents, dont des interviews par canton. L'équipe a démontré une bonne maîtrise de la méthodologie, de la présentation des résultats aussi bien que des recommandations. Cependant, le programme initial de mise en valeur a été modifié ; commerçants et bureaucrates se sont partagés de vastes domaines mis en valeur grâce aux techniques modernes (tracteurs) et une importante main d'œuvre agricole (plusieurs dizaines d'ouvriers agricoles).

Henri Raulin a écrit une note à ce sujet Michel Keïta et moi avons attiré l'attention de la plus haute autorité administrative, c'est-à-dire le Préfet de Niamey. J'ai évité de donner à qui que ce soit l'occasion de croire que je défendais "notre terroir". En effet le slogan "La terre à celui qui la cultive" faisait sourire les propriétaires fonciers ; l'incitation au retour à la terre pour assurer l'auto suffisance alimentaire, très favorablement accueillie par la bourgeoisie (commerçante, bureaucratique), était une source supplémentaire d'accumulation. Les éleveurs aussi étaient attirés par l'amélioration des possibilités d'abreuvement dans une zone connue, même si personne ne pouvait dire avec certitude à qui appartenait le bétail.

Notre équipe avait donc eu raison d'insister sur le déplacement, vers le sud, de la limite de la réserve, dans la mesure où il fallait concilier exploitation et réserve. 
Après avoir perdu le privilège d'être "sociologue national", j'ai appris pour la première fois que je n'étais point sociologue. Instantanément, je vis là une intoxication provenant d'un homme politique. Par honnêteté, cette hypothèse fut vite écartée. Ce pouvait être l'opinion d'un conseiller technique, "expert en études des structures mentales", reprise par la "voix de son maître". Mais, et si c'était la différence de formation? La licence en sociologie comprenait quatre certificats d'études supérieures, dont trois obligatoires (sociologie générale, psychologie sociale, économie politique et sociale) et une à option (civilisation musulmane, linguistique africaine). J'ignore les autres combinaisons possibles, en particulier les améliorations ultérieures. Mais je pense que le sociologue doit nécessairement avoir le sens de la diachronie et de la relativité. Mes travaux ne contiennent donc aucune recette. Il m'a toujours semblé que savoir rédiger un rapport exploitable par le demandeur est un art difficile. Mais je reconnais que cela peut susciter des frustrations qui doivent être situées à leur juste niveau. J'ai pensé aussi que cela pouvait être le signe de l'appétit créé par la réalisation d'études à la demande des services locaux et surtout des organismes internationaux, c'est à dire par le filon "expertise".

Celui qui a pu bénéficier d'un enseignement diversifié, solide, en un mot d'une meilleure formation, résultant elle-même d'une évolution normale, sera jugé à l'aide de deux critères au moins :

- capacité à étudier les sociétés africaines, et à proposer des explications acceptables en termes de sociologie africaine (recherche fondamentale),

- contribution à l'amélioration du cadre de vie des populations africaines (recherche appliquée, "expertise")

Vers 1972, Michel Keita et moi proposions la formation de l'association des sociologues : la troisième génération y décèle l'intention de l'embrigader dans le Parti Progressiste Nigérien Rassemblement Démocratique Africain, PPN-RDA. Une telle association naîtra nécessairement, sans Michel hélas! Car il faut apprendre à discuter avec des disciples de l'empirisme anglo-saxon et du structuralisme germano-français, surtout quand on veut faire de la sociologie fondée sur l'histoire et les cultures africaines.

Vers 1982, je déclare ignorer à quoi correspond la "tribu" au Niger et exige l'utilisation du terme "nationalité ". Le problème est donc de préciser quelle sociologie nous voulons. Si le rôle se réduit à ignorer le nombre actuel de langues nationales, combattre leur utilisation, imposer l'Etat-nation aux peuples africains, n'être ni participant-observateur, ni observateur-participant dans les conférences nationales, alors je préfère être un non-sociologue.

\section{BIBLIOGRAPHIE}

1. Problèmes généraux 
Boubacar Ly. 1989 Problèmes épistémologiques et méthodologiques des sciences sociales en Afrique, Dakar, UNESCO/CODESRIA.

Michel Keita 1982 Situation et perspective de la recherche en sciences sociales au Niger, Niamey, IRSH, $10 \mathrm{p}$.

Pierre-Philippe Rey. juin 1991 L'anthropologie et l'engagement de la politique à la pratique, Paris, Cahier du GEMDEV, $\mathrm{n}^{\circ}$ 18, pp. 71-83.

André Salifou février 1971 Colloque sur le rôle des sciences sociales et humaines dans le développement, Lomé, 24-27, $11 \mathrm{p}$ (rapport de mission).

Hamidou Arouna Sidikou. juillet-août 1983 Recherche comparative et trans-culturelle sur les facteurs sociaux affectant le développement scientifique endogène. Le cas du Niger, Niamey, IRSH/UNESCO, 108 p.

Albert Wright. 22-27 juin 1973 Consultation régionale de l'UNESCO en vue de la préparation de CASTAFRICA, Abidjan, $8 \mathrm{p}$ (rapport de mission).

2. Rapports

2.1. Expert associé

Suzanne Bernus. juin 1968 Les problèmes de la zone industrielle de Niamey, Niamey, CNRSH, 29 p. annexes.

Issaka Doulaye et al. août 1972 Enquête de base à Tillabéri. Etude du milieu, Niamey, CNRSH, 62 p.

Michel Keïta. juillet 1974 Enquête sur l'acceptabilité des produits de la Sotramil, Niamey, IRSH.

Michel Keïta et al. 1976 Etude de Say. Rapport intérimaire, Niamey, IRSH/PNUD.

Michel Keïta et al. 1977 a) Etude de Say. Rapport final, Niamey, IRSH/PNUD.

Michel Keïta et al. 1977b) Etude de Say. Interviews par canton, 5 volumes, Niamey, IRSH/PNUD.

Dr. Charles Pidoux. juin 1967 Les migrations internes et internationales au Niger de 1960 à 1967, Niamey, CNRSH, $17 \mathrm{p}$.

2.2 Expert

mars 1966 Rapport sur la cuvette de Kirkissoye (Vallée du Niger), Niamey, IFAN-CNRSH, 40 p.

février 1968 Note sur la situation des personnes âgées de 50 ans et plus dans la Commune de Niamey, Niamey, CNRSH, 18 p. nomenclatures des professions, tableaux.

1968 Recherche et Développement. Le projet de mise en valeur des cuvettes de Kutukale et Karma en pays songhay, Niamey, CNRSH, Etudes Nigériennes, $n^{\circ} 24,145$ p. annexes.

juillet 1971 Rapport de mission dans la zone du Projet de développement de la production animale et des ressources en eau dans l'est du Niger, Niamey, CNRSH, 15 p.

1976 Prospection socio-économique pour l'implantation de puits, in Keïta et al., pp. 109-176.

1977 Démographie légère, in Keïta et al. 1977a, pp 143-196.

\section{NOTES}

1.Ce texte devait être présenté oralement lors du colloque sur "Les sciences sociales et l'expertise en Afrique", organisé par l'APAD, en Février 1994 à Bamako. 
2.Il s'agit d'un gigantesque projet de Barrage hydraulique, situé sur le fleuve Niger dans la région de Tillabéri.

3.J'ai effectué des études secondaire au Soudan (Mali actuel) au lycée Terrasson de Fougères.

AUTEUR

DIOULDÉ LAYA

CELTHO/OUA Niamey 